\title{
MEDIUM RESOLUTION NMR AT 20 MHz: POSSIBILITIES AND CHALLENGES
}

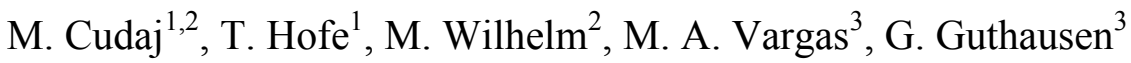 \\ ${ }^{1}$ PSS, In der Dalheimer Wiese 5, 55120 Mainz, \\ ${ }^{2}$ Institute for Chemical Technology and Polymer Chemistry, KIT, 76131 Karlsruhe, \\ ${ }^{3}$ SRG 10-2, Institute for Mechanical Engineering and Mechanics, KIT, 76131 Karlsruhe, \\ Germany
}

\section{INTRODUCTION}

Detailed studies of compositions, for example in food and oil industries ${ }^{1-4}$, are of great importance to guarantee a high and constant quality and well defined properties of a product. Many applications still need expensive analytical techniques like high resolution NMR (Nuclear Magnetic Resonance) to determine each component's concentration like the marginal amounts of acids, colorants or polyphenols ${ }^{5}$ in wine analysis ${ }^{6}$ or small amounts of special fatty acids in virgin olive oils ${ }^{7}$. Moreover high field NMR delivers valuable information for characterizing the botanical origin or for investigating the age of a natural product like vinegar ${ }^{8,9}$.

Up to now, low field NMR instruments in the range of $1-60 \mathrm{MHz}$ are almost exclusively applied in relaxometry studies ${ }^{10,11}$, which proved to be a powerful method, for example, to characterize the movement and redistribution of water within soils on the basis of $T_{2}$ relaxation times of the water proton signal. However, there have been very few investigations on the ability of low field instruments delivering spectral information, concerning chemical shifts and $J$-coupling of the studied sample.

In contrast to relaxometry methods for determination of water and oil content ${ }^{12-17}$, prerequisite for frequency resolved NMR proton spectra is a sufficient spectral resolution of the NMR spectrometer in the range of $0.2 \mathrm{ppm}$ full width half maximum (FWHM) ${ }^{18}$ and frequency or $\boldsymbol{B}_{0}$ field stability. The general feasibility of NMR spectroscopy with adequate resolution at low magnetic fields have already been shown by different groups ${ }^{19-22}$. The technique is named medium resolution NMR (MR-NMR) in the following.

Apart from composition determination via MR-NMR, processes like fermentation, ripening or encapsulation of active agents are of interest. Reactant concentrations can principally be followed as a function of time allowing the study of reaction kinetics.

Moreover, a question which has to be addressed is the way how raw data are processed; the possibilities include integration or the statistical chemometric approach. Whether or not the limited spectral resolution and sensitivity at low magnetic fields can be "compensated" more effectively by "classical" data processing (integration and subsequent linear regression) or multivariate data analysis (chemometrics) is studied on two models. The 
ability of chemometrics as a tool for analysis of high field NMR spectra of mixtures has already been shown ${ }^{23}$.

\section{INSTRUMENTATION}

Apart from a modified Bruker the minispec mq20 for on-line spectroscopy, the instrument used for these measurements was a medium resolution (MR-NMR) prototype from Bruker (Bruker BioSpin $\mathrm{GmbH}$, Rheinstetten, Germany), which works at a proton $\left({ }^{1} \mathrm{H}\right)$ Larmor frequency of about $20.1 \mathrm{MHz}$ and is based on a permanent magnet ${ }^{18}$. The magnet temperature is stabilized at $40^{\circ} \mathrm{C}$ within $\pm 0.001{ }^{\circ} \mathrm{C}$. With passive, mechanical shimming the homogeneity of the magnet system is in the order of $1 \mathrm{ppm}$ for a cylindrical sample volume of about ID $4 \mathrm{~mm} \times 12 \mathrm{~mm}$. Furthermore, the spectrometer features an electronic shim system with 12 channels with adjustable currents in the range of $\pm 100 \mathrm{~mA}$. With this planar shim system a field homogeneity of around $0.1 \mathrm{ppm}$ (FWHM), sufficient for MRNMR, can be achieved.

The MR-NMR prototype was equipped with a home built probe with a quality factor $Q=140$ for sample tubes with an outer diameter of $5 \mathrm{~mm}$ and with a $13 \mathrm{~mm}$ sensitive region along $y$. The optimum $\pi / 2$ flip angle was obtained at $3 \mu$ s (transmitter power of about $5 \mathrm{~W}$ ). Data acquisition with application of FIR (finite impulse response) digital filters is provided, involving an enormous data reduction factor due to on-the-fly oversampling. This leads to a significant $S / N$ advantage once the improvement in homogeneity leads to longer FID (free induction decay) decay times in the order of several hundreds of ms.

\section{IDENTIFICATION AND QUANTIFICATION}

Conventional high field NMR spectroscopy is frequently used for identification and quantification of (unknown) substances. However, its spectral resolution is far better than in MR-NMR. In the following, some dedicated examples show the use of MR-NMR for identification and quantification.

\subsection{Fatty acids composition}

A variety of different oils, like sunflower or olive oil, were analyzed by ${ }^{1} \mathrm{H}-\mathrm{NMR}$ spectroscopy with a $500 \mathrm{MHz}$ NMR system ${ }^{7}$. NMR spectroscopy provided a possible alternative to conventional chromatographic methods for determining the chemical composition of oils.

With the $20 \mathrm{MHz}$ spectrometer, spectra of rape oil and sunflower oil were acquired, both fats containing large amounts of unsaturated fatty acids. In contrast, a spectrum of Palmin ${ }^{\circledR}$ was recorded; a fat that contains "only" saturated fatty acids like palmitin acid due to hydrogenation. Figure 1 shows spectra of the three different oils respectively solid fat (molten at $40{ }^{\circ} \mathrm{C}$, which is the sample temperature due to the tempering of the magnet at $40^{\circ} \mathrm{C}$ ). All spectra were generated by recording 128 scans and folding the practically noise free FID with Gauss-Lorentzian functions. The recycle delay time amounted to $1 \mathrm{~s}$ while the acquisition time was chosen as $0.67 \mathrm{~s}$. 


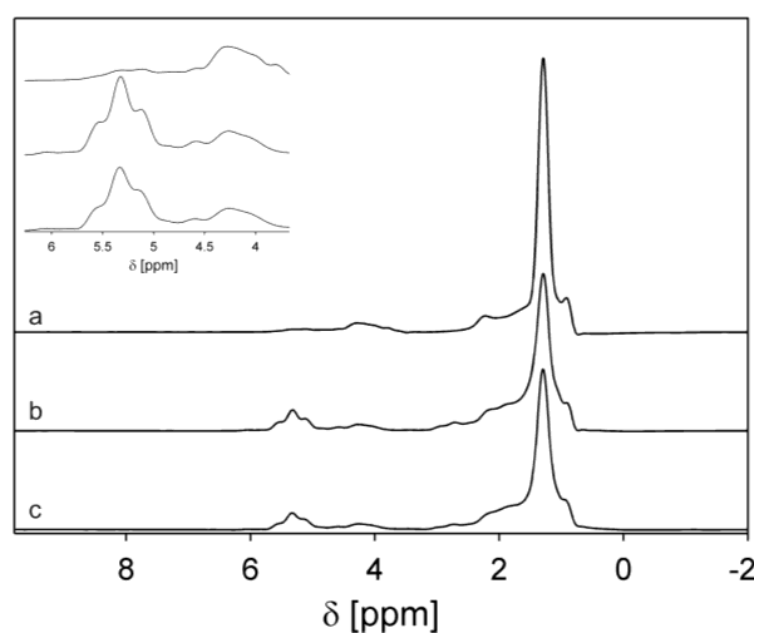

Figure 1: ${ }^{1}$ H-spectra of Palmin $\circledR(a)$, sunflower oil (b) and rape oil (c). The spectra show relatively unspecific lines in the aliphatic spectral region (1-2 ppm). The significant peaks of carbon double bonds, which appear at $5.3 \mathrm{pm}$ in spectra $b$ and $c$, are $a$ factor of 5 less in spectrum a.
In the aliphatic spectral region (1-2 ppm) the different fat compositions do not differ significantly. In contrast the olefinic region is characteristic for each sample. Obviously, the significant peak of carbon double bonds, which appears around $5.3 \mathrm{ppm}$ in the spectra of rape oil and sunflower oil, is clearly diminished in the spectrum of Palmin ${ }^{\circledR}$. The spectral fraction of unsaturated fatty acids is estimated to $8.1 \%$ (rape oil), $9.8 \%$ (sun flower oil) and $2.2 \%$ (Palmin $\AA)$, which is in good agreement with the compositional expectation.

\subsection{Pyrolysis oils from renewable natural resources}

A field of large scientific interest is currently the energy generation from plants, especially from straw of food plants and woods. A major problem is the high and varying water content during biomass gasification which complicates the measurement and control process. A fast and reliable technique is needed which is additionally robust against environmental influences.

It has to be proven, that MR-NMR is a well-suited technique, by its ability to measure the water content of the pyrolysis oils. Figure 2 shows the MR-NMR spectra of oils with different botanical origin and pyrolysis processes. Apart from the water peak around $4.6 \mathrm{ppm}$, also the chemical composition of the oils varies significantly, which can be seen most obviously in the aliphatic region. Depending on the viscosity of the oils, the spectral
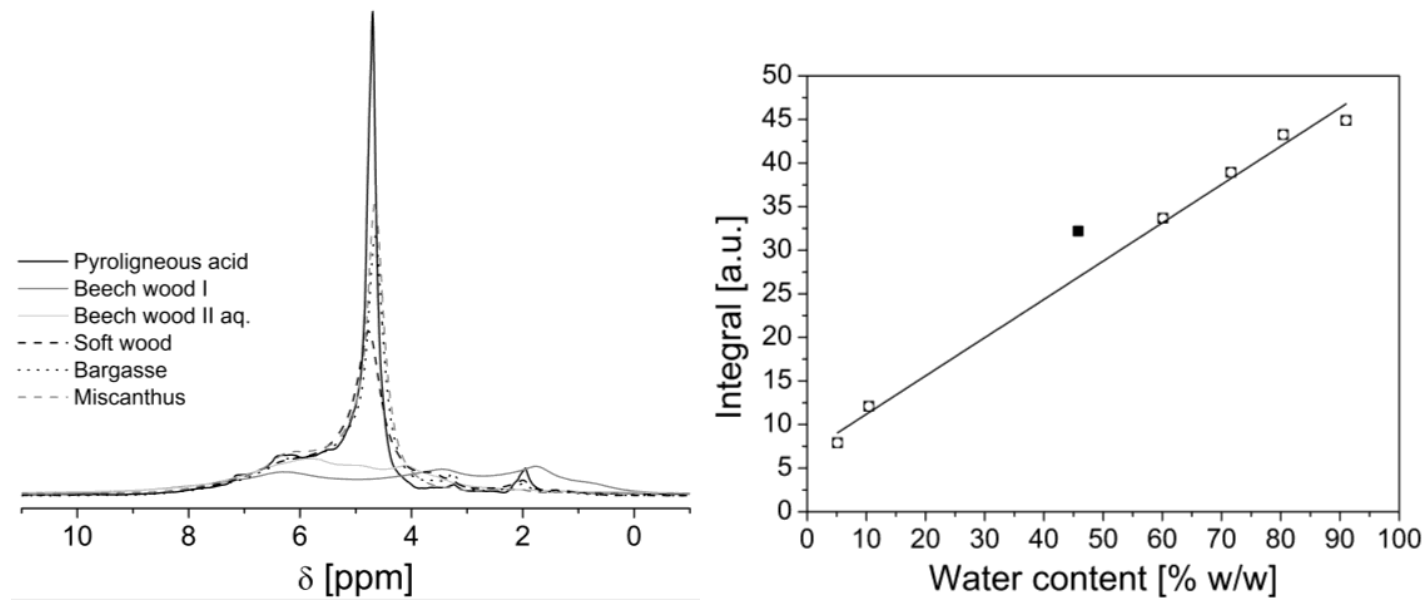

Figure 2: The water content of various pyrolysis oils can be clearly identified in the ${ }^{I} H$-NMR spectra. In spite of their different chemical composition which is also observable over the spectral range, a correlation between the integral of the water peak around 4.6 ppm and alternatively determined water content can be established. The coefficient of determination amounts to 0.99. 
signatures vary in their line width so that a chemical composition determination is not easily possible. However, the water line is relatively pronounced. Its integration was correlated with the water content determined by wet chemical procedures. A satisfying agreement was obtained with a coefficient of determination $R^{2}=0.99(0.97$, when considering the outlier).

The spectra were measured averaging 4 scans with a repetition time of $10 \mathrm{~s}$, a dwell time of $163.5 \mu \mathrm{s}$ and 8k acquisition data points. During data processing, zero-filling by a factor of two was applied.

\subsection{Concentration Determination in Binary Compounds}

Currently, wine quality analysis is often performed by wet chemical procedures (including e. g. $\mathrm{Cr}^{6+}$ ) or infrared methods. Comparing the efforts of wet chemical methods with those required by low field NMR spectroscopy, MR-NMR could be a valuable tool for reducing efforts in ethanol determination. Compared with IR, the higher selectivity of NMR could be of advantage. However, accuracy and reproducibility have to be proven.

Figure 3 shows the spectra of a ternary ethanol water isopropanol mixture (a, 30 vol. $\%$ ethanol, 30 vol. $\%$ isopropanol) and a binary ethanol water mixture $(b, 14$ vol.\% ethanol) at $20 \mathrm{MHz}$. The spectra were recorded with 8 scans for sufficient signal to noise ratio, $8 \mathrm{k}$ data points and a zero filling factor of 4 in order to assure an adequate resolution. The FID acquisition time amounts to about $1.34 \mathrm{~s}$, while the recycle delay time was $25 \mathrm{~s}$ guaranteeing complete recovery of the magnetization $\left(5 T_{1}\right)$. To

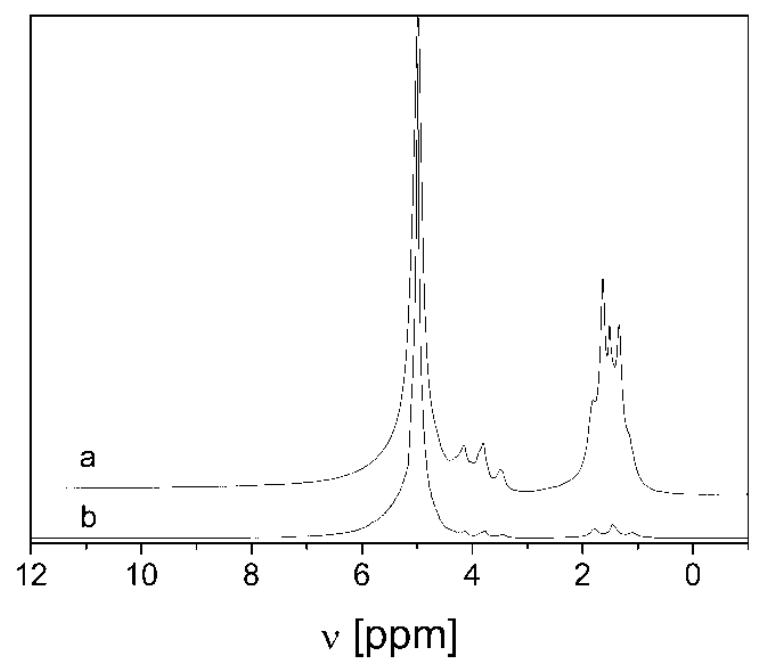

Figure 3: Spectra of ternary ethanol water isopropanol mixture $(30$ vol.\% ethanol, 30 vol.\% isopropanol, a) and of binary ethanol water mixture (14 vol.\% ethanol, b). The spectra are highly overlapping due to $B_{0}$ inhomogeneity and the relatively large $J$-coupling compared to chemical shift. Clearly distinguishable are the $\mathrm{CH}_{3}$-lines at 12 ppm, the $\mathrm{CH}_{2}$-groups show up between 3 and 4 ppm. The dominant peak in the spectra is due to water.

avoid further peak broadening due to magnetic field drifts and fluctuations, the spectra were frequency-corrected during data processing.

Compared to commonly known high field spectra of these substances, the lower relation of chemical shift to $J$-coupling at $20 \mathrm{MHz}$ leads to different shapes and often considerable broadening of single, chemical shift separated peaks. Both the larger $B_{0}$ inhomogeneity and the larger $J$-coupling (in $\mathrm{ppm}$ ) lead to highly overlapping signals. The question to be answered is to which extent larger peak widths and insufficient baseline separation of MRNMR spectra affect the analysis of binary and ternary compounds in ,classical” and multivariate data processing. A second question is which data processing method results in reproducible and accurate concentration determination. Apart from classical integration of specific peaks, multivariate data analysis is applied in form of PLS (partial least squares) regression (e.g., introduction into PLS-R ${ }^{24}$ ).

An accurate determination of concentrations from NMR spectra requires absolutely reproducible baselines, which are preferentially completely flat. Therefore, a section wise base line correction was performed. Integration limits were chosen in a way that the 
influence of neighbouring lines of the other moieties was minimal; on the other hand the sections should be still meaningful for the concentration determination.

PLS regression was accomplished using the TOMCAT tool ${ }^{25}$. It is based on Matlab ${ }^{\circledR}$ and is an open source program ${ }^{25}$. Cross validation was performed leaving out two samples of 36 calibration samples $(+4$ test set samples) with a maximum of 1000 iterations resulting in the calibration model in figure 4 (left). The large number of iterations was chosen because of increased numerical stability of the model. The intercept as well as the slope of the resulting linear regression equation $y=0.99997 x+0.0006$ indicate a good correlation between "balance-determined" $(x)$ and "measured" values $(y)$ for ethanol concentration. Additionally, figure 4 shows the results for three test samples (right, $\mathrm{x}$ ) in the calibration model as well as a residual plot. The optimum for the calibration was found at a rank of 10 . When comparing the values of additional $200 \mathrm{MHz}$ data with MR-NMR data (Table 1), one can assert that the correlation coefficient $R$ as well as the model characterizing errors $R M S$ (root mean square error), $R M S E C V$ (root mean square error of cross validation) and $R M S E P$ (root mean square value of prediction) are in the same range for high and low field measurements. For the quality of measurement and model, the RMSEP, indicating an average absolute prediction error, is the most important value, which is in the range of 0.1 to $0.2 \%$. It has to be compared with the required accuracy which is $<0.25 \mathrm{vol} \%$ for German wines at the moment. This result implies the possibility of determining ethanol contents, e. g., in wine analysis by MR-NMR spectroscopy with the help of multivariate data processing.
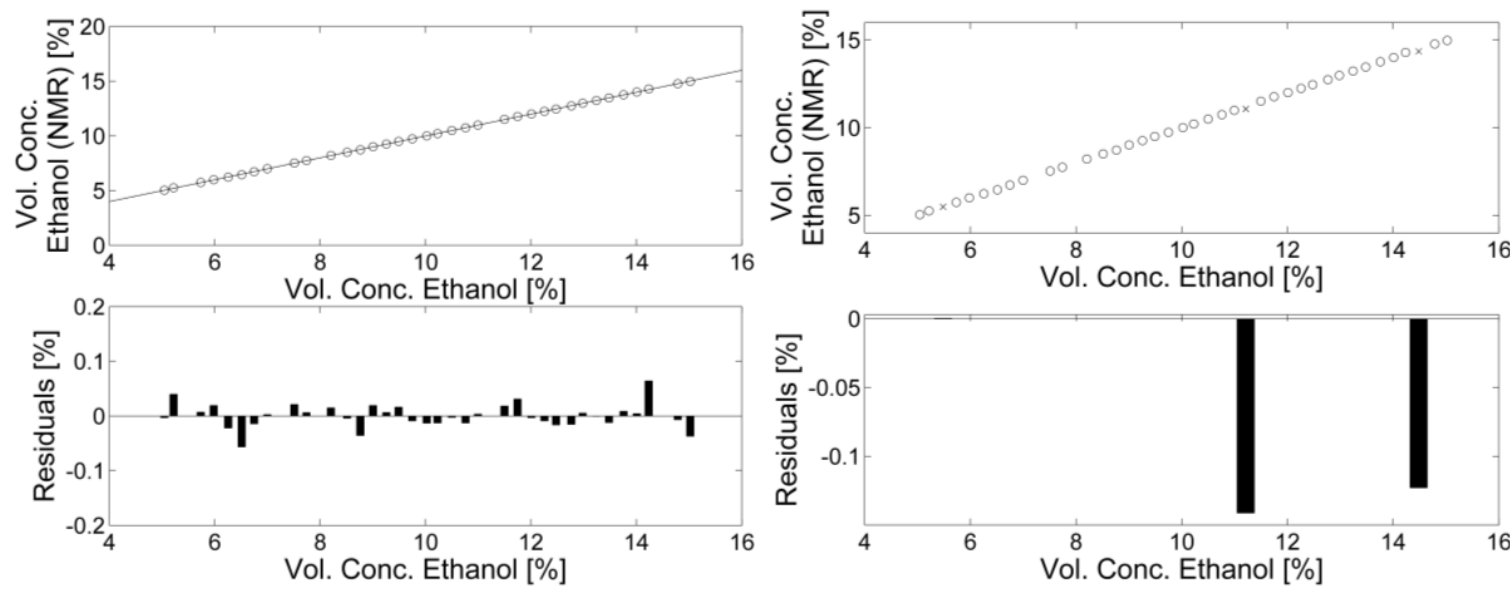

Figure 4: Calibration (left, o) and test set validation (right, x) for ethanol quantification, obtained by applying a PLS regression to the ${ }^{I} H-M R-N M R$ data. The ethanol determination is therefore regarded as being quantitative with rather small errors, which are indicated by the residuals for each sample in the calibration and the test sets, respectively. The coefficient of determination in the correlation plot amounts to $R^{2}=0.9999, R M S E P=0.11$ vol. $\%$.

Table 1: Regression results of $20 \mathrm{MHz}$ and $200 \mathrm{MHz}$ data, processed by applying PLS-R and integration.

\begin{tabular}{|c|c|c|c|c|}
\hline & $\begin{array}{l}20 \mathrm{MHz} \\
\text { PLS }\end{array}$ & $\begin{array}{l}200 \mathrm{MHz} \\
\text { PLS }\end{array}$ & $\begin{array}{l}20 \mathrm{MHz} \text {, } \\
\text { integration }\end{array}$ & $\begin{array}{l}200 \mathrm{MHz}, \\
\text { integration }\end{array}$ \\
\hline $\begin{array}{l}\text { Linear } \\
\text { regression }\end{array}$ & $\begin{array}{ll}y=0.99995 x+ & 0.0006 \\
(0.997 x+0.029) & \end{array}$ & $y=0.998 x+0.019$ & $y=0.661 x+1.546$ & $y=0.825 x+2.260$ \\
\hline$R$ & $0.99997(0.9986)$ & 0.999 & 0.947 & 0.955 \\
\hline$R M S$ & $0.021(0.157)$ & 0.128 & 0.669 & 0.767 \\
\hline RMSECV & $0.041(0.165)$ & 0.207 & - & - \\
\hline RMSEP & $0.108 *(0.198)$ & 0.214 & 1.150 & 1.357 \\
\hline
\end{tabular}


The conventional quantification method in NMR spectroscopy is the integration of specific peaks. The assumption is that the integral of a well separated line can be directly correlated to the number of spins of a specific moiety, in this case of ethanol or water. Spectra were analyzed so that the integral of the $\mathrm{CH}_{3}$-peak, which corresponds to half of the ${ }^{1} \mathrm{H}$ spins of ethanol, was taken as a measure for ethanol concentration. The volume concentration was calculated by consideration of molar mass and mass density of water and ethanol, without regarding the fact of volume contraction due to negative partial volumes when mixing water and ethanol. Baseline corrections were performed aiming for an accurate determination of the methyl group integral and of the entire integral due to higher precision in the choice of integration limits. As in multivariate data analysis, the same three of the 40 samples were left out from the linear regression model as they were needed as test samples and calculation of RMSEP. Neither linear regression nor mean prediction error could reach the quality of their chemometric analogues (Table 1). The "classical" data procedure is clearly less accurate and reliable compared to multivariate PLS-R analysis and could not reach required accuracy limits $(R M S E P<0.25$ vol.\%). Baseline drifts, imperfect shim currents, insufficient automated phase corrections and others led to this impreciseness, which finally favours chemometric methods for the investigation of binary compounds in the $5-15 \%$ range of absolute ethanol content.

Concluding, the spectroscopic measurement of binary mixtures of ethanol and water by MR-NMR spectroscopy, combined with chemometric data processing, shows its potential in quantitative determination of relatively high concentrated substances. Compared with $200 \mathrm{MHz}$, the accuracy is in the same order of magnitude for the high concentration samples.

\section{ON-LINE PROCESS CONTROL}

In process analytics material changes are of great interest. Processes like ripening, encapsulation of active agents and chemical reactions should preferentially be followed online. Analytical techniques like infrared spectroscopy and ultrasound measurements partially fulfil this need for time-resolved analyst concentration monitoring. An alternative could be MR-NMR with its inherent high chemical selectivity. First examples are described in literature ${ }^{17,20-22}$.

In this work the modified minispec mq $20^{19}$ was used to monitor the reaction progress in an emulsion polymerization ${ }^{26}$. This reaction type is of special interest in food processing science because of the above mentioned encapsulation possibility of active agents in droplets. Exemplarily, polymerization process of butyl-acrylate was monitored. From the NMR point of view the essential reaction is a carbon reduction from a $\mathrm{sp}^{2}$ - to a $\mathrm{sp}^{3}-$ hybridized state. Additionally, the sensitivity of transverse relaxation towards molecular mobility has to be considered.

In figure 5 the spectrum at reaction start is shown. As butyl-acrylate has $\mathrm{sp}^{2}$-hybridized carbon atoms, a line around $6.3 \mathrm{ppm}$ can be observed. The decrease of its intensity is a direct measure for the reaction progress. Additionally, residual $\mathrm{H}_{2} \mathrm{O}$ and aliphatic components can be clearly distinguished. The line at the right is due to the reference in the probe, which is used as an independent measure for frequency correction and reproducibility check. This is necessary because of magnetic field drifts on the short- and long term scale. The reaction was performed in $\mathrm{D}_{2} \mathrm{O}$ because of the dominant $\mathrm{H}_{2} \mathrm{O}$ peak. 

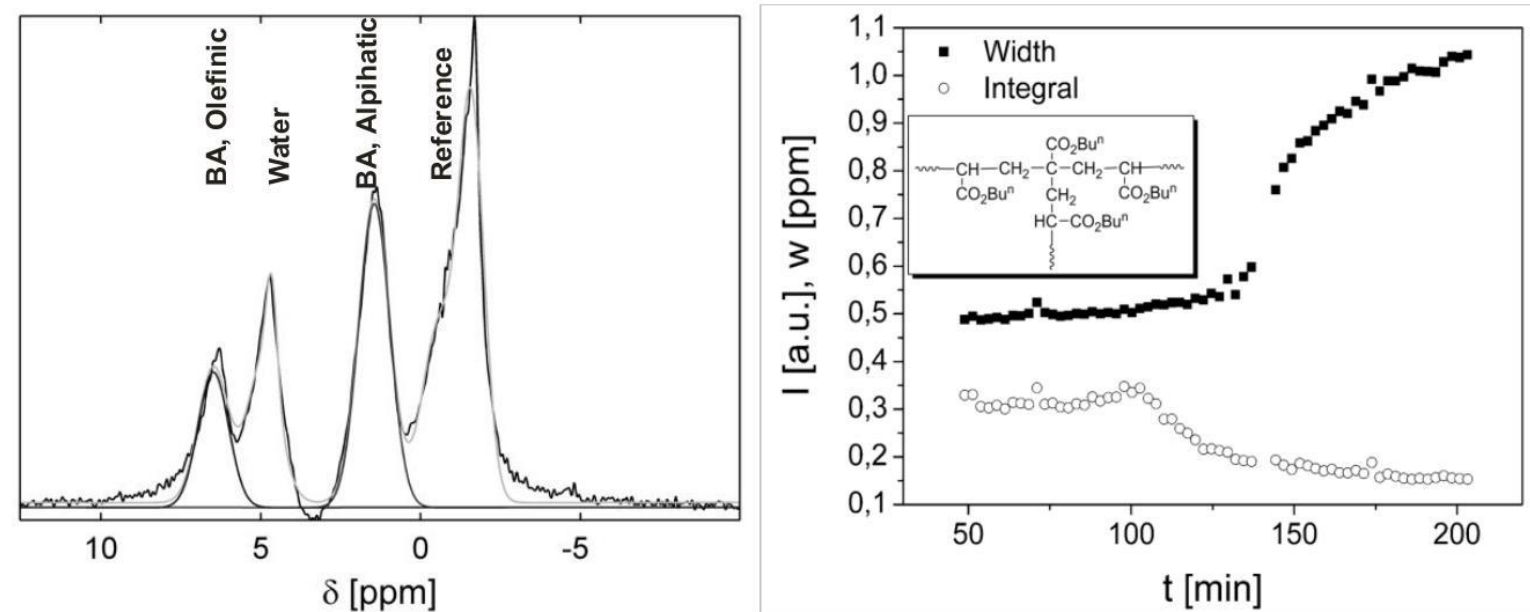

Figure 5: Typical spectrum of butyl-acrylate in $\mathrm{D}_{2} \mathrm{O}$ (left) as measured with a modified mq20. The olefinic line at $6.3 \mathrm{ppm}$ decreases in amplitude whereas in the aliphatic region an increase of line width and amplitude can be observed. The line around $4.6 \mathrm{ppm}$ is mainly due to residual $\mathrm{H}_{2} \mathrm{O}$, the peak at $-2.0 \mathrm{ppm}$ comes from reference sample in the probe. On the right hand side the integral of the olefinic region and the line width in the aliphatic region are shown as a function of reaction time.

All spectra were fitted in a MATLAB ${ }^{\circledR}$ routine resulting in graphs for intensities and widths ${ }^{26}$. The significant parameters are the intensity of the olefinic peak and the line width in the aliphatic region. These two parameters can be described by kinetic models, revealing typical rate constants for the emulsion polymerization ${ }^{26}$. Please note the time shift between the start of integral decrease and width increase, which is caused by different physical phenomena reflected by the two NMR spectral parameters.

The reaction can also be followed when performed in $\mathrm{H}_{2} \mathrm{O}$. However, due to the limited spectral resolution of the current instrumentation, the error bars of the olefinic intensity are rather large. Nevertheless, the line width in the aliphatic region can be used for numerical analysis for determination of the kinetic coefficients.

\section{CONCLUSIONS}

MR ${ }^{1} \mathrm{H}-\mathrm{NMR}$ spectra $(20 \mathrm{MHz}$, resolution $<0.2 \mathrm{ppm})$ can be a real alternative to conventional analytical tools, even more in combination with multivariate data analysis. When comparing the MR-NMR spectra with those measured at a 10 times higher field strength on samples with sufficiently high concentrations, it could be shown that data processing by multivariate analysis tools is an essential element that can improve prediction ability, accuracy and reproducibility of an analyzed system. The limiting factors are for highly concentrated samples not the signal to noise ratio nor the spectral resolution, but artefacts like phase and baseline errors as well as spurious signals.

In addition to concentration and identification studies, MR-NMR spectroscopy can also be used as a chemically sensitive method in reaction studies and control. It provides detailed insight into the reaction kinetics. 


\section{Acknowledgements}

M. C. and G. G. would like to thank Bruker BioSpin GmbH (especially A. Kamlowski and D. C. Maier) for allocation of the MR-NMR prototype spectrometer as well as Bruker BioSpin development team for valuable discussions and speedy help. The 'Shared Research Group 10-2' received financial support by the 'Concept for the future' of Karlsruhe Institute of Technology within the framework of the German Excellence Initiative. Additional support was given by FhG ICT and DFG, which are also highly appreciated. Financial support by the Investitions- und Strukturbank Rhineland-Palatinate (ISB) $\mathrm{GmbH"}$ is gratefully acknowledged. M. V. acknowledges the financial support by the DAAD.

\section{References}

Please consider also the references in the cited publications.

1 I. F. Duarte, A. N. Barros, C. U. Almeida, M. Spraul, A. M. Gil, J. Agric. Food Chem., 2004, 52, 1031.

2 M. R. Monteiro, A. R. P. Ambrozin, L. M. Lião, E. F. Boffo, E. R. Pereira-Filho, A. G. Ferreira, J. Am. Oil. Chem. Soc., 2009, 86, 581 .

3 M. R. Monteiro, A. R. P. Ambrozina, M. da Silva Santos, E. F. Boffo, E. R. Pereira-Filho, L. M. Lião, A. G. Ferreira, Talanta, 2009, 78, 660.

4 M. R. Monteiro, A. R. P. Ambrozin, L. M. Liao, E. F. Boffo, L. A. Tavares, M. M. C. Ferreira, A. G. Ferreira, Energy \& Fuels, 2009, 23, 272.

5 F. H. Larsen, F. van den Berg, S. B. Engelsen, J. Chemometrics, 2006, 20, 198.

6 G. E. Pereira, J.-P. Gaudillere, C. van Leeuwen, G. Hilbert, O. Lavialle, M. Maucourt, C. Deborde, A. Moing, D. Rolin, J. Agric. Food Chem., 2005, 53, 6382.

7 C. Fauhl, F. Reniero, C. Guillou, Magn. Reson. Chem., 2000, 38, 436.

8 R. Consonni, L.R. Cagliani, F. Benevelli, M. Spraul, E. Humpfer, M. Stocchero, Analytica Chimica Acta, 2008, 611, 31 .

9 G. J. Martin, C. Guillou, M. L. Martin, M. T. Cabanis, Y. Tep and J. Aerny, J. Agric. Food Chem., 1988, 36, 316.

10 T. R. Todoruk, M. Litvina, A. Kantzas, C. H. Langford, Environ. Sci. Technol., 2003, 37, 2878.

11 C. A. Toussaint, F. Médale, A. Davenel, B. Fauconneau, P. Haffray, S. Akoka, J. Sci. Food Agric., 2002, 82, 173.

12 H. T. Pedersen, L. Munck, S. B. Engelsen, J. Am. Oil. Chem. Soc., 2000, 77, 1069.

13 J. Brùndum, L. Munck, P. Henckel, A. Karlsson, E. Tornberg, S. B. Engelsen, Meat Science, 2000, 55, 177.

14 S. M. Jepsen, H. T. Pedersen, S. B Engelsen, J Sci. Food Agric., 1999, 79, 1793.

15 A. Guthausen, G. Guthausen, H. Todt, W. Burk, A. Kamlowski, D. Schmalbein, J. Am. Oil. Chem. Soc., 2004, 81, 727.

16 G. Guthausen, J. König, A. Kamlowski, Bruker Spin Report, 2004, 154, 41.

17 A. Nordon, P.J. Gemperline, C.A. McGill, and D. Littlejohn, 2001, 73, 4286.

18 M. Cudaj, G. Guthausen, A. Kamlowski, D. Maier, T. Hofe, M. Wilhelm, to appear in Nachrichten aus der Chemie, November 2010.

19 G. Guthausen, A. von Garnier, R. Reimert, Applied Spectroscopy, 2009, 63, 1121.

20 A. Nordon, A. Diez-Lazaro, C. W. L. Wong, C. A. McGill, D. Littlejohn, M. Weerasinghe, D. A. Mamman, M. L. Hitchman, J. Wilkie, Analyst, 2008, 133, 339.

21 T. W. Skloss, A. J. Kim, J. F. Haw, Analytical Chemistry, 1994, 66, 536.

$22 \mathrm{http}: / /$ www.qualion-nmr-com

23 H. Winning, F. H. Larsen, R. Bro, S. B. Engelsen, J. Magn. Reson., 2008, 190, 26.

24 P. Geladi and B. R. Kowalski, Analytica Chimica Acta, 1986, 185, 1.

25 M. Daszykowski, S. Serneels, K. Kaczmarek, P. van Espen, C. Croux, and B. Walczak, Chemom. Intell. Lab. Syst., 2007, 85, 269.

26 M. A. Vargas, M. Cudaj, K. Hailu, K. Sachsenheimer, and G. Guthausen, Macromolecules, 2010, 43, 5561 . 